\title{
Heterosis and Combining Ability Evaluation for Yield, Quality, and Fruit Fly Resistance in Muskmelon
}

\author{
Saroj Rolania ${ }^{1}$ * and M.S. Fageria ${ }^{2}$ \\ ${ }^{1}$ Defence Research and Development Organization, Delhi-110054, India \\ ${ }^{2}$ Genemics and Biotechnology Forest and Conservation Genomics and Biotechnology Lab, \\ Faculty of Forestry and Environmental management, University of New Brunswick, \\ Tweeddale Centre, 1350 Regent Street, Fredericton NB E3C 2C6, Canada \\ *Corresponding author
}

Keywords

Muskmelon, Heterosis, gca, sca, Fruit yield, Fruit fly, Resistance

Article Info

Accepted:

08 June 2018

Available Online:

10 July 2018

\section{A B S T R A C T}

The objective of this study was to breed muskmelon hybrids possessing higher TSS, flesh, and rind thickness, early and total yields; lower seed cavity; and resistance to fruit fly. Eight genetically diverse parents viz., Kesar, exotic collections (EC-2, EC-3, EC-4, EC-5), and indigenous collections (GP-210, GP-211, GP-141) were crossed in a diallel fashion excluding reciprocals. The resulting $28 \mathrm{~F}_{1}$ 's were evaluated with their parents in completely randomized block design with three replications under open field conditions. A high positive and significant commercial heterosis was shown by a couple of crosses. The analysis of variance revealed presence of considerable genetic variability for all the characters studied. Estimates of general combining ability (gca) and specific combining ability (sca) were highly significant. Parent GP-210 and GP-211 were best general combiners for vine length, number of fruits per hill and TSS; GP-211 and GP-141 for days to first fruit harvest; EC-5 and EC-3 for fruit weight; EC-2 and Kesar for small size of seed cavity, higher flesh thickness, rind thickness and resistance to fruit fly. The two best crosses were Kesar x EC-2 and EC-3 x GP-211. Kesar x EC-2 was superior in fruit weight, yield, size of seed cavity, flesh and rind thickness, shelf life and resistance to fruit fly. EC$3 \times$ GP-211 took lesser days to harvest first fruit and recorded higher fruit weight, yield, rind thickness and had least incidence of fruit fly.

\section{Introduction}

South Africa is considered as primary centre of origin of muskmelon (Cucumis melo L.) while India is considered as secondary centre of diversity. In a tropical country like India, juicy dessert fruits like muskmelon and watermelon are considered as best thrust quenchers during the arid summer months. In spite of its economic importance and availability of considerable genetic diversity very little improvement especially in relation to quality, shelf life, and resistance to fruit fly has been done in this crop. The exploitation of heterosis is a potential approach for the amelioration of all those crops where sufficient variability exists and hybrid seed can be conveniently produced. It had, 
therefore been most successfully used approach in the crops where cross-pollination frequently occurs. A speedy improvement can be brought about in muskmelon by assessing the genetic variability and its exploitation through hybrid breeding. Muskmelon is highly cross-pollinated crop (Swarup, 1991) and shows considerable amount of heterosis. Hybrids in muskmelon have been valued for varied reasons. They include earliness, fruit uniformity, higher yield, quick and easy introduction of dominant genes, resistant to different diseases, etc. Being dessert fruit, quality parameters, especially TSS, flesh thickness, texture, colour and higher sugar content are the important one. Round fruits with orange thick flesh and tough/netted rind suitable for long distance transportation are preferred in the market. Earliness is of greater importance, as market prices for such a crop are exorbitant and they can ultimately lead to higher net returns to the growers. An F1 hybrid of muskmelon yields more than the standard cultivars (Munger, 1942; Spasov, 1963; Foster, 1967; Lippert and Hall, 1972a; More and Seshadri, 1980, Mishra and Seshadri, 1985; Dhaliwal and Lal, 1996 and Munshi and Verma, 1997). Systematic breeding programmes carried out in different parts of the world have brought about excellent hybrid varieties with good fruit qualities for local and distant markets and resistance to diseases, but the efforts made in this direction in muskmelon in India are meager. The growers of north India are still not happy with the existing varieties of muskmelon especially due to their low yield potential, poor keeping quality, and lower sweetness. Therefore the objective of this study was to develop hybrids of muskmelon combining all these desirable characteristics.

\section{Materials and Methods}

The large number of germplasm of muskmelon is being maintained at
Agricultural Research Station, Durgapura, Jaipur, India. On the basis of yield, quality, and resistance characteristics, eight genetically diverse genotypes of muskmelon including Kesar, exotic collections (EC-2, EC-3, EC-4, and EC-5), and endogenous collections (GP210, GP-211 and GP-141) were selected for this study. These eight genotypes were crossed in a diallel fashion excluding reciprocals. The resulting $28 \mathrm{~F}_{1} \mathrm{~s}$ and their eight parents along with a control (commercial hybrid Abhijeet) were grown in a randomized block design with three replications. The seeds were sown in furrows maintaining $200 \mathrm{~cm}$ spacing between rows and $60 \mathrm{~cm}$ between hills, each row containing of ten plants. After every two lines, one line of Durgapura Madhu, a cultivar highly susceptible to fruit fly was planted to create natural epiphytotic in the field. All recommended cultural and management practices were followed to raise a healthy crop. Observations on various characters in each replication were recorded timely. All the observations were recorded on five randomly selected plants in each treatment and average was taken. Vine length was measured from the main axis to the highest tip of plant and recorded in meters at the end of crop season. The number of days taken to first fruit harvest was counted from the date of sowing the crop to first fruit harvest. The total weight of first three harvested fruits in kilogram $(\mathrm{kg})$ was divided by three to obtain the average weight. To count number of marketable fruits per hill, the marketable fruits harvested periodically were counted and the final data (as total number of marketable fruits) consisted of the pooling of such periodical harvestings till the end of the experiment were summed up to get the data on fruit yield per plant. Five mature fruits from each line were taken to measure their seed cavity $(\mathrm{cm})$ by an ordinary scale. The average size of seed cavity was calculated. The fruits used to measure the size of seed cavity were also utilized to calculate rind thickness $(\mathrm{cm})$. The rind of cut fruits was 
peeled out carefully and measured with the help of an ordinary scale. The same fruits were used to record the data on flesh thickness. For TSS, the flesh of five ripe fruits was crushed separately and their juice passed through a double layer of fine mesh cheese cloth. A drop of juice was placed on the plate of Abbe's Hand Refractometer (0-32 \%) and the reading was noted. A mean of five readings was taken in every case. To calculate Shelf life (days), three healthy fruits per line were kept at room temperature to ascertain the shelf-life of fruits. In order to determine the degree of fruit damage by the fruit fly (Dacus cucurbitae Coq.), the total number of damaged and undamaged fruits were counted separately in every picking. The difference between healthy and damaged fruits was made on the basis of presence of punctures made by larvae on damaged fruits. The percentage of damage was calculated by the given formula

Per cent infested fruits $=$

Number of infested fruits

Total number of fruits

The nature of resistance or susceptibility of all the lines under study was determined by a definite rating system formulated and used by Nath (1966) as presented in table A.

The qualitative characters of harvested fruits of muskmelon viz., fruit shape, rind colour, fruit skin texture, skin hardiness, presence or absence of sutures, flesh texture, flesh colour and fruit flavour were evaluated by a panel of five judges and the fruits were categorized accordingly.

Heterosis was calculated as per Fonsceca and Patterson (1968) and combining ability as per Griffing (1956). Heterosis was calculated over better parent and standard check. Depending upon the desirability of the character, first or second parent was treated as better parent.

\section{Results and Discussion}

Analysis of variance (Table 1) revealed significant differences among parents and hybrids for all the characters indicating sufficient genetic variability among genotypes for exploitation of heterosis. The magnitude of variance due to gca were found to be greater than that of sca for all the characters, suggesting the pre-dominance of additive gene effects.

The highest desirable heterobeltiosis for yield and its component characters was observed in order of rind thickness $(96.67 \%)$, shelf life $(96.43 \%)$, yield/hill $(81.97 \%)$, flesh thickness $(60.34 \%)$, fruits/hill $(36.03 \%)$, fruit weight $(30.00 \%)$, vine length $(22.22 \%)$, TSS $(19.23 \%)$, fruit fly incidence $(-41.72 \%)$, size of seed cavity $(-22.78 \%)$ and days to first fruit harvest $(-8.52 \%)$. The gca effects were found to be highly significant. The parent GP-211 had desirable gca effects for vine length, days taken to first fruit harvest, fruits per hill, fruit yield, and TSS. The cross EC-3 x GP-211 had high per se performance for fruit yield per hill and also showed desirable sca effects for rind thickness, and shelf life. A perusal of sca effects revealed 10 hybrids for vine length, 5 for days to first fruit harvest, 10 for fruit weight, 11 for fruit yield, 6 for size of seed cavity, 10 for rind thickness, 10 for flesh thickness, 2 for TSS, 15 for shelf life, and 10 for incidence of fruit fly exhibited significant sca effects (Table 1).

The highest heterobeltiosis for vine length was recorded in the cross EC-3 x EC-5 (22.22\%). Earliness is economically desirable for seasonal marketing demands. The hybrid EC-3 $x$ EC-5 exhibited heterosis in a desirable direction over better parent as well as standard check. Highest negative sca effects were observed in the crosses EC-3 x EC-5 and GP$210 \times$ GP-211. Both the $F_{1}$ 's took about 5-10 days lesser for the fruit maturity than their 
respective parents. These crosses were derived from good $\mathrm{x}$ good combiners, indicating additive type of gene action. It seems that one should more focus on developing inbred lines first, so that the expected earliness may be achieved in resulting $\mathrm{F}_{1}$ 's. These results are in consonance with those of Chadha and Nandpuri (1980b), More and Seshadri (1980), Mishra and Seshadri (1985), Randhawa and Singh (1990), McCreight et al., (1993), Munshi and Verma (1997) and Choudhary (2002). The cross EC-2 x EC-3 exhibited maximum heterosis for fruit weight $(30.00 \%)$ over better parent (Table 1). It was significantly higher in eight crosses compared to standard check. Improvement in fruit weight through heterosis breeding in muskmelon has been reported by Dixit and Kalloo (1983), Randhawa and Singh (1990), Dhaliwal and Lal (1996), Munshi and Verma (1998), Choudhary et al., (2003), Feyzian et al., (2009) and Shasikumar and Pitchaimuthu 2016. Hence, heterobeltiosis can be exploited for having high fruit weight in muskmelon. Parents EC-5, EC-3, EC-2, Kesar, GP-211 and GP-141 were good general combiners. The crosses EC-2 x EC-3 and GP-210 x GP-211 had shown highest sca effects. These $\mathrm{F}_{1}$ 's derived from good $\mathrm{x}$ good and poor $\mathrm{x}$ good combining parents, showing importance of both gca and sca. The hybrids EC-3 x EC-4 and EC-3 x GP-210 exhibited heterosis in a desirable direction over better parent as well as standard check for number of fruits per hill. Crosses EC-3 x EC-4 and EC-3 x GP-210 had highest sca effects. These $\mathrm{F}_{1}$ 's derived from poor $\mathrm{x}$ poor and poor $\mathrm{x}$ good combining parents.

Yield improvement is the primary goal in all the crop improvement programmes. Thirteen crosses showed significantly positive heterobeltiosis for fruit yield per hill. Seven crosses had recorded higher fruit yield over standard hybrid Abhijeet. The maximum heterosis over better parent was found to be 81.97 per cent in cross EC-3 x EC-4. This cross had also shown higher number of marketable fruits per hill and therefore, it could be considered as one of the best $F_{1}$ 's. The appreciable amount of heterosis for this trait has also been reported in literature (Nandpuri et al., 1974b; Pandey and Kalloo, 1976; Chadha and Nandpuri, 1980b; More and Seshadri, 1985; Randhawa and Singh, 1990; Dhaliwal and Lal, 1996; Munshi and Verma, 1997 and 1999 and Choudhary, 2002) (Table 2-7).

Table.1 Analysis of variance for different characters

\begin{tabular}{|c|c|c|c|c|c|c|}
\hline \multirow[t]{2}{*}{ Characters } & \multicolumn{6}{|c|}{ Mean Sum of Squares } \\
\hline & $\begin{array}{l}\text { Replications } \\
\text { d.f. } 2\end{array}$ & $\begin{array}{l}\text { Treatments } \\
35\end{array}$ & $\begin{array}{l}\text { Error } \\
70\end{array}$ & $\begin{array}{l}\text { GCA } \\
7\end{array}$ & $\begin{array}{l}\text { SCA } \\
28\end{array}$ & $\begin{array}{l}\text { Error } \\
70\end{array}$ \\
\hline Vine length & 0.011 & $0.752 * *$ & 0.025 & $0.897 * *$ & $0.089 * *$ & 0.008 \\
\hline Days to first fruit harvest & 19.313 & $63.339 * *$ & 7.073 & $63.930 * *$ & $10.412 * *$ & 2.358 \\
\hline $\begin{array}{l}\text { Av. weight of first three } \\
\text { harvested fruits }\end{array}$ & 0.002 & $0.091 * *$ & 0.004 & $0.112 * *$ & $0.010 * *$ & 0.0013 \\
\hline No. of marketable fruits/hill & 0.055 & $4.281 * *$ & 0.177 & $4.906^{* *}$ & $0.557^{* *}$ & 0.059 \\
\hline Yield/hill & 0.117 & $2.933 * *$ & 0.123 & $1.094 * *$ & $0.949 * *$ & 0.041 \\
\hline Size of seed cavity & 0.116 & $1.546 * *$ & 0.136 & $1.162 * *$ & $0.354 * *$ & 0.045 \\
\hline Rind thickness & 0.009 & $0.034 * *$ & 0.001 & $0.019 * *$ & $0.009 * *$ & 0.0004 \\
\hline Flesh thickness & 0.005 & $1.180 * *$ & 0.042 & $1.032 * *$ & $0.234 * *$ & 0.014 \\
\hline TSS & 0.468 & $7.833 * *$ & 1.090 & $10.765 * *$ & 0.573 & 0.363 \\
\hline Shelf life & 0.022 & $3.908 * *$ & 0.064 & $3.091 * *$ & $0.855^{* *}$ & 0.022 \\
\hline Severity of viruses & 0.242 & $48.028 * *$ & 0.626 & $49.128 * *$ & $7.730 * *$ & 0.209 \\
\hline Incidence of fruit fly & 0.441 & $80.321 * *$ & 1.683 & 73.719** & $15.037 * *$ & 0.561 \\
\hline
\end{tabular}


Table.2 Vine length, days taken to first fruit harvest and average weight of first three harvested fruits of parents, $\mathrm{F}_{1}$ 's and heterosis (over better parent and check) in muskmelon

\begin{tabular}{|c|c|c|c|c|c|c|c|c|c|}
\hline \multirow[b]{2}{*}{ Parents and $F_{1}$ 's } & \multicolumn{3}{|c|}{ Vine length } & \multicolumn{3}{|c|}{ Days taken to first fruit harvest } & \multicolumn{3}{|c|}{$\begin{array}{l}\text { Average weight of first three } \\
\text { harvested fruits }\end{array}$} \\
\hline & $\begin{array}{l}\text { Mean } \\
(\mathrm{m})\end{array}$ & $\begin{array}{l}\text { Per cent } \\
\text { heterosis } \\
\text { over BP }\end{array}$ & $\begin{array}{c}\text { Per cent } \\
\text { increase/ } \\
\text { decrease } \\
\text { over hybrid } \\
\text { Abhijeet }\end{array}$ & $\begin{array}{l}\text { Mean } \\
\text { (days) }\end{array}$ & $\begin{array}{c}\text { Per cent } \\
\text { heterosis } \\
\text { over BP }\end{array}$ & $\begin{array}{c}\text { Per cent } \\
\text { increase/de } \\
\text { crease over } \\
\text { hybrid } \\
\text { Abhijeet }\end{array}$ & $\begin{array}{c}\text { Mean } \\
(\mathrm{kg})\end{array}$ & $\begin{array}{c}\text { Per cent } \\
\text { heterosis } \\
\text { over BP }\end{array}$ & $\begin{array}{c}\text { Per cent } \\
\text { increase/de } \\
\text { crease over } \\
\text { hybrid } \\
\text { Abhijeet }\end{array}$ \\
\hline Kesar & 1.80 & - & - & 79.80 & - & - & 0.91 & - & - \\
\hline EC-2 & 2.50 & - & - & 88.37 & - & - & 1.00 & - & - \\
\hline EC-3 & 2.10 & - & - & 81.00 & - & - & 0.95 & - & - \\
\hline EC-4 & 2.00 & - & - & 88.30 & - & - & 0.65 & - & - \\
\hline EC-5 & 1.50 & - & - & 88.70 & - & - & 1.10 & - & - \\
\hline GP-210 & 3.00 & - & - & 91.17 & - & - & 0.66 & - & - \\
\hline GP-211 & 2.93 & - & - & 83.80 & - & - & 0.95 & - & - \\
\hline GP-141 & 2.60 & - & - & 82.40 & - & - & 1.00 & - & - \\
\hline Kesar x EC-2 & 2.31 & -7.60 & 5.00 & 80.51 & 0.88 & -0.85 & 1.20 & $20.00 *$ & $20.00 *$ \\
\hline Kesar x EC-3 & 2.00 & -4.76 & -9.09 & 76.30 & -4.39 & $-6.03 *$ & 1.10 & $15.79 *$ & 10.00 \\
\hline Kesar x EC-4 & 2.13 & 6.67 & -3.03 & 81.83 & 2.55 & 0.78 & 0.93 & 2.57 & -7.00 \\
\hline Kesar x EC-5 & 1.87 & 3.70 & $-15.15^{*}$ & 88.00 & $10.28 *$ & $8.37 *$ & 1.20 & 9.09 & $20.00 *$ \\
\hline Kesar x GP-210 & 3.45 & $15.00 *$ & $56.82 *$ & 91.17 & $14.24 *$ & $12.28 *$ & 0.72 & $-20.59 *$ & $-28.00 *$ \\
\hline Kesar x GP-211 & 3.10 & 5.68 & $40.91 *$ & 77.20 & -3.26 & -4.93 & 1.02 & 7.02 & 1.67 \\
\hline Kesar x GP-141 & 2.60 & 0.00 & $18.18^{*}$ & 76.00 & -4.76 & $-6.40 *$ & 1.11 & $11.33^{*}$ & $11.33^{*}$ \\
\hline EC-2 x EC-3 & 2.33 & -6.67 & 6.06 & 83.50 & 3.09 & 2.83 & 1.30 & $30.00 *$ & 30.00* \\
\hline EC-2 $\times$ EC-4 & 2.23 & $-10.67 *$ & 1.52 & 84.40 & -4.42 & 3.94 & 0.90 & -10.00 & -10.00 \\
\hline EC-2 x EC-5 & 2.13 & $-14.67 *$ & -3.03 & 85.80 & -2.90 & $5.67 *$ & 1.20 & 9.09 & $20.00 *$ \\
\hline EC-2 x GP-210 & 3.17 & 5.56 & $43.94 *$ & 87.23 & -1.28 & $7.43^{*}$ & 0.83 & $-17.00 *$ & $-17.00 *$ \\
\hline EC-2 x GP-211 & 2.80 & -4.55 & $27.27 *$ & 83.23 & -0.68 & 2.50 & 0.90 & -10.00 & -10.00 \\
\hline EC-2 x GP-141 & 2.50 & -3.85 & $13.64 *$ & 82.13 & -0.32 & 1.15 & 1.00 & 0.00 & 0.00 \\
\hline EC-3 x EC-4 & 2.33 & 11.11 & 6.06 & 79.80 & -1.48 & -1.72 & 1.00 & 5.26 & 0.00 \\
\hline EC-3 x EC-5 & 2.57 & $22.22 *$ & $16.67^{*}$ & 75.70 & $-6.54^{*}$ & $-6.77 *$ & 1.31 & $19.09^{*}$ & 31.00* \\
\hline EC-3 x GP-210 & 3.10 & 3.33 & $40.91 *$ & 84.33 & 4.12 & 3.85 & 0.78 & $-17.89 *$ & $-22.00 *$ \\
\hline EC-3 x GP-211 & 3.01 & 2.61 & $36.82 *$ & 74.67 & $-7.82 *$ & $-8.05^{*}$ & 1.20 & $26.32 *$ & $20.00 *$ \\
\hline EC-3 x GP-141 & 2.73 & 5.13 & $24.24 *$ & 74.10 & $-8.52^{*}$ & $-8.74 *$ & 1.07 & 7.33 & 7.33 \\
\hline EC-4 x EC-5 & 1.95 & -2.67 & -11.52 & 85.33 & -3.36 & 5.09 & 0.95 & $-13.64^{*}$ & -5.00 \\
\hline EC-4 x GP-210 & 3.23 & 7.67 & $46.82^{*}$ & 86.10 & -2.49 & $6.03 *$ & 0.65 & -1.52 & $-35.00 *$ \\
\hline EC-4 x GP-211 & 3.22 & $9.66^{*}$ & $46.21 *$ & 79.50 & -5.13 & -2.09 & 0.90 & -5.26 & -10.00 \\
\hline EC-4 x GP-141 & 2.63 & 1.15 & $19.55^{*}$ & 84.40 & 2.43 & 3.94 & 0.90 & -10.00 & -10.00 \\
\hline EC-5 x GP-210 & 3.07 & 2.22 & $39.39^{*}$ & 85.40 & -3.72 & 5.17 & 0.81 & $-26.36^{*}$ & $-19.00 *$ \\
\hline EC-5 x GP-211 & 3.00 & 2.27 & $36.36^{*}$ & 80.20 & -4.30 & -1.23 & 1.17 & 6.36 & $17.00 *$ \\
\hline EC-5 x GP-141 & 2.60 & 0.00 & $18.18^{*}$ & 83.00 & 0.73 & 2.22 & 1.10 & 0.00 & 10.00 \\
\hline GP-210 x GP-211 & 3.34 & $11.44 *$ & $51.97 *$ & 79.90 & -4.65 & -1.60 & 0.96 & 1.05 & -4.00 \\
\hline GP-210 x GP-141 & 3.15 & 5.11 & $43.33^{*}$ & 81.50 & -1.09 & 0.37 & 0.80 & $-20.00 *$ & $-20.00 *$ \\
\hline GP-211 x GP-141 & 2.94 & 0.34 & $33.79 *$ & 75.80 & $-8.01^{*}$ & $-6.65^{*}$ & 0.99 & -1.00 & -1.33 \\
\hline Abhijeet (Check) & 2.20 & - & - & 81.20 & - & - & 1.00 & - & - \\
\hline S Ed \pm & 0.130 & & & 2.170 & & & 0.050 & & \\
\hline CD at $5 \%$ & 0.255 & & & 4.32 & & & 0.101 & & \\
\hline
\end{tabular}

* Significant at $\mathrm{p}=0.05$ 
Table.3 Number of marketable fruits per hill, fruit yield per hill and size of seed cavity of parents, $\mathrm{F}_{1}$ 's and heterosis (over better parent and check) in muskmelon

\begin{tabular}{|c|c|c|c|c|c|c|c|c|c|}
\hline \multirow[b]{2}{*}{ Parents and $F_{1}$ 's } & \multicolumn{3}{|c|}{ No. of marketable fruits per hill } & \multicolumn{3}{|c|}{ Fruit yield per hill } & \multicolumn{3}{|c|}{ Size of seed cavity } \\
\hline & Mean & $\begin{array}{l}\text { Per cent } \\
\text { heterosis } \\
\text { over BP }\end{array}$ & $\begin{array}{l}\text { Per cent } \\
\text { increase/de } \\
\text { crease over } \\
\text { hybrid } \\
\text { Abhijeet }\end{array}$ & $\begin{array}{c}\text { Mean } \\
(\mathrm{kg})\end{array}$ & $\begin{array}{l}\text { Per cent } \\
\text { heterosis } \\
\text { over BP }\end{array}$ & $\begin{array}{c}\text { Per cent } \\
\text { increase/de } \\
\text { crease over } \\
\text { hybrid } \\
\text { Abhijeet }\end{array}$ & $\begin{array}{c}\text { Mean } \\
(\mathrm{cm})\end{array}$ & $\begin{array}{l}\text { Per cent } \\
\text { heterosis } \\
\text { over BP }\end{array}$ & $\begin{array}{c}\text { Per cent } \\
\text { increase/de } \\
\text { crease over } \\
\text { hybrid } \\
\text { Abhijeet }\end{array}$ \\
\hline Kesar & 3.80 & - & - & 3.50 & - & - & 5.00 & - & - \\
\hline EC-2 & 4.60 & - & - & 4.60 & - & - & 4.50 & - & - \\
\hline EC-3 & 4.31 & - & - & 4.07 & - & - & 7.50 & - & - \\
\hline EC-4 & 5.44 & - & - & 3.60 & - & - & 6.00 & - & - \\
\hline EC-5 & 4.36 & - & - & 4.80 & - & - & 6.50 & - & - \\
\hline GP-210 & 8.00 & - & - & 5.30 & - & - & 6.80 & - & - \\
\hline GP-211 & 6.10 & - & - & 5.60 & - & - & 6.30 & - & - \\
\hline GP-141 & 6.33 & - & - & 5.80 & - & - & 6.00 & - & - \\
\hline Kesar x EC-2 & 5.75 & $25.00 *$ & -4.17 & 6.90 & $50.00 *$ & $15.00 *$ & 4.30 & -4.44 & $-21.82 *$ \\
\hline Kesar x EC-3 & 5.45 & $26.45^{*}$ & -9.17 & 6.00 & $47.54 *$ & 0.00 & 7.30 & $46.00 *$ & 32.73* \\
\hline Kesar x EC-4 & 6.42 & $18.01 *$ & 7.00 & 6.00 & $66.67 *$ & 0.00 & 5.80 & $16.00 *$ & 5.45 \\
\hline Kesar x EC-5 & 4.92 & 12.92 & $-17.94 *$ & 5.90 & $22.92 *$ & -1.67 & 6.60 & $32.00 *$ & $20.00 *$ \\
\hline Kesar x GP-210 & 7.43 & $-7.12 *$ & $23.83^{*}$ & 5.20 & -1.89 & $-13.33^{*}$ & 6.30 & $26.00 *$ & $14.55 *$ \\
\hline Kesar x GP-211 & 6.67 & 9.29 & 11.11 & 6.00 & 7.14 & 0.00 & 5.90 & $18.00 *$ & 7.27 \\
\hline Kesar x GP-141 & 5.36 & $-15.37 *$ & -10.67 & 6.00 & 3.45 & 0.00 & 6.17 & $23.33^{*}$ & 12.12* \\
\hline EC-2 $\times$ EC-3 & 4.92 & 6.96 & $-18.00 *$ & 6.40 & $39.13^{*}$ & 6.67 & 7.10 & $57.78^{*}$ & 29.09* \\
\hline EC-2 x EC-4 & 5.56 & 2.21 & -7.33 & 5.00 & 8.70 & $-16.67 *$ & 5.97 & $32.59 *$ & 8.48 \\
\hline EC-2 x EC-5 & 4.25 & -7.61 & $-29.17^{*}$ & 5.10 & 6.25 & $-15.00^{*}$ & 6.50 & $44.44 *$ & $18.18 *$ \\
\hline EC-2 x GP-210 & 7.15 & $-10.67 *$ & $19.11^{*}$ & 5.90 & $11.32 *$ & -1.67 & 5.70 & $26.67 *$ & 3.64 \\
\hline EC-2 x GP-211 & 6.67 & 9.34 & 11.17 & 6.00 & 7.14 & 0.00 & 5.60 & $24.44 *$ & 1.82 \\
\hline EC-2 x GP-141 & 5.04 & $-20.42 *$ & $-16.00^{*}$ & 5.00 & $-13.79 *$ & $-16.67 *$ & 5.83 & $29.63 *$ & 6.06 \\
\hline EC-3 x EC-4 & 7.40 & $36.03 *$ & $23.33^{*}$ & 7.40 & $81.97 *$ & $23.33^{*}$ & 6.00 & 0.00 & 9.09 \\
\hline EC-3 x EC-5 & 5.42 & $24.31 *$ & -9.67 & 7.10 & $47.92 *$ & $18.33^{*}$ & 6.27 & -3.59 & 13.94* \\
\hline EC-3 x GP-210 & 8.53 & 6.62 & $42.17^{*}$ & 6.60 & $24.53^{*}$ & $10.00 *$ & 7.00 & 2.94 & $27.27 *$ \\
\hline EC-3 x GP-211 & 6.32 & 3.61 & 5.33 & 7.60 & $35.71 *$ & $26.67 *$ & 5.60 & $-11.11^{*}$ & 1.82 \\
\hline EC-3 x GP-141 & 5.63 & $-11.11^{*}$ & -6.17 & 6.00 & 3.45 & 0.00 & 6.43 & 7.22 & $16.97 *$ \\
\hline EC-4 x EC-5 & 4.85 & -10.85 & $-19.17^{*}$ & 4.60 & -4.17 & $-23.33^{*}$ & 6.33 & 5.56 & $15.15 *$ \\
\hline EC-4 x GP-210 & 8.32 & 4.00 & $38.67^{*}$ & 5.40 & 1.89 & $-10.00^{*}$ & 4.63 & $-22.78^{*}$ & $-15.76^{*}$ \\
\hline EC-4 x GP-211 & 6.12 & 0.33 & 2.00 & 5.50 & -1.79 & -8.33 & 5.80 & -3.33 & 5.45 \\
\hline EC-4 x GP-141 & 5.53 & $-12.68^{*}$ & -7.83 & 4.90 & $-15.52^{*}$ & $-18.33^{*}$ & 5.90 & -1.67 & 7.27 \\
\hline EC-5 x GP-210 & 7.23 & $-9.62 *$ & $20.50 *$ & 5.90 & $11.32^{*}$ & -1.67 & 6.90 & 6.15 & $25.45 *$ \\
\hline EC-5 x GP-211 & 6.32 & 3.61 & 5.33 & 7.50 & $33.93^{*}$ & $25.00 *$ & 5.50 & $-12.70^{*}$ & 0.00 \\
\hline EC-5 x GP-141 & 5.45 & $-13.95^{*}$ & -9.17 & 6.00 & 3.45 & 0.00 & 6.50 & 8.33 & $18.18^{*}$ \\
\hline $\begin{array}{l}\text { GP-210 x GP- } \\
211\end{array}$ & 7.08 & $-11.50 *$ & $18.00^{*}$ & 6.80 & $21.43^{*}$ & $13.33^{*}$ & 6.40 & 1.59 & $16.36^{*}$ \\
\hline $\begin{array}{l}\text { GP-210 x GP- } \\
141\end{array}$ & 7.53 & -5.87 & $25.50 *$ & 6.00 & 3.45 & 0.00 & 5.90 & -1.67 & 7.27 \\
\hline $\begin{array}{l}\text { GP-211 x GP- } \\
141\end{array}$ & 5.61 & $-11.42^{*}$ & -6.50 & 5.50 & -5.17 & -8.33 & 6.20 & 3.33 & $12.73^{*}$ \\
\hline $\begin{array}{l}\text { Abhijeet } \\
\text { (Check) }\end{array}$ & 6.00 & - & - & 6.00 & - & - & 5.50 & - & - \\
\hline S Ed \pm & 0.340 & & & 0.290 & & & 0.300 & & \\
\hline CD at $5 \%$ & 0.684 & & & 0.569 & & & 0.598 & & \\
\hline
\end{tabular}


Table.4 Flesh thickness, rind thickness, and shelf life of parents, $\mathrm{F}_{1}$ 's and heterosis (over better parent and check) in muskmelon

\begin{tabular}{|c|c|c|c|c|c|c|c|c|c|}
\hline \multirow[b]{2}{*}{ Parents and $F_{1}$ 's } & \multicolumn{3}{|c|}{ Flesh thickness } & \multicolumn{3}{|c|}{ Rind thickness } & \multicolumn{3}{|l|}{ Shelf life } \\
\hline & $\begin{array}{l}\text { Mean } \\
(\mathrm{cm})\end{array}$ & $\begin{array}{l}\text { Per cent } \\
\text { heterosis } \\
\text { over BP }\end{array}$ & $\begin{array}{c}\text { Per cent } \\
\text { increase/de } \\
\text { crease over } \\
\text { hybrid } \\
\text { Abhijeet }\end{array}$ & $\begin{array}{l}\text { Mean } \\
(\mathrm{cm})\end{array}$ & $\begin{array}{l}\text { Per cent } \\
\text { heterosis } \\
\text { over BP }\end{array}$ & $\begin{array}{c}\text { Per cent } \\
\text { increase/de } \\
\text { crease over } \\
\text { hybrid } \\
\text { Abhijeet }\end{array}$ & $\begin{array}{l}\text { Mean } \\
\text { (days) }\end{array}$ & $\begin{array}{l}\text { Per cent } \\
\text { heterosis } \\
\text { over BP }\end{array}$ & $\begin{array}{c}\text { Per cent } \\
\text { increase/de } \\
\text { crease over } \\
\text { hybrid } \\
\text { Abhijeet }\end{array}$ \\
\hline Kesar & 3.60 & - & - & 0.50 & - & - & 5.00 & - & - \\
\hline EC-2 & 4.30 & - & - & 0.40 & - & - & 4.90 & - & - \\
\hline EC-3 & 1.73 & - & - & 0.30 & - & - & 2.80 & - & - \\
\hline EC-4 & 3.00 & - & - & 0.30 & - & - & 3.14 & - & - \\
\hline EC-5 & 2.80 & - & - & 0.30 & - & - & 3.90 & - & - \\
\hline GP-210 & 2.07 & - & - & 0.20 & - & - & 1.44 & - & - \\
\hline GP-211 & 1.93 & - & - & 0.20 & - & - & 1.90 & - & - \\
\hline GP-141 & 2.50 & - & - & 0.40 & - & - & 4.80 & - & - \\
\hline Kesar x EC-2 & 4.13 & -3.88 & $33.33^{*}$ & 0.60 & $20.00 *$ & 5.26 & 6.50 & $30.00 *$ & 30.00* \\
\hline Kesar x EC-3 & 3.10 & $-13.89^{*}$ & 0.00 & 0.52 & 4.00 & -8.77 & 4.90 & -2.00 & -2.00 \\
\hline Kesar x EC-4 & 3.60 & 0.00 & $16.13^{*}$ & 0.50 & 0.00 & $-12.28^{*}$ & 5.30 & 6.00 & 6.00 \\
\hline Kesar x EC-5 & 3.50 & -2.78 & $12.90^{*}$ & 0.53 & 6.00 & -7.02 & 5.00 & 0.00 & 0.00 \\
\hline Kesar x GP-210 & 2.73 & $-24.07 *$ & $-11.83^{*}$ & 0.48 & -4.00 & $-15.79 *$ & 6.00 & $20.00 *$ & $20.00 *$ \\
\hline Kesar x GP-211 & 3.30 & -8.33 & 6.45 & 0.50 & 0.00 & $-12.28 *$ & 5.20 & 4.00 & 4.00 \\
\hline Kesar x GP-141 & 3.77 & 4.63 & $21.51^{*}$ & 0.56 & $11.33^{*}$ & -2.34 & 5.50 & $10.00 *$ & $10.00 *$ \\
\hline EC-2 x EC-3 & 4.00 & -6.98 & $29.03^{*}$ & 0.43 & 7.50 & $-24.56^{*}$ & 5.00 & 2.04 & 0.00 \\
\hline EC-2 $x$ EC-4 & 2.60 & $-39.53^{*}$ & $-16.13^{*}$ & 0.47 & $17.50^{*}$ & $-17.54^{*}$ & 4.80 & -2.04 & -4.00 \\
\hline EC-2 x EC-5 & 2.77 & $-35.66^{*}$ & -10.75 & 0.45 & 12.50 & $-21.05^{*}$ & 5.10 & 4.08 & 2.00 \\
\hline EC-2 x GP-210 & 2.03 & $-52.71^{*}$ & $-34.41^{*}$ & 0.41 & 1.67 & $-28.65^{*}$ & 4.90 & 0.00 & -2.00 \\
\hline EC-2 x GP-211 & 3.00 & $-30.23^{*}$ & -3.23 & 0.43 & 7.50 & $-24.56^{*}$ & 5.30 & 8.16 & 6.00 \\
\hline EC-2 x GP-141 & 2.50 & $-41.86^{*}$ & $-19.35^{*}$ & 0.49 & $22.50 *$ & $-14.04 *$ & 5.20 & 6.12 & 4.00 \\
\hline EC-3 x EC-4 & 2.53 & $-15.56^{*}$ & $-18.28 *$ & 0.48 & $60.00 *$ & $-15.79^{*}$ & 4.20 & $33.76^{*}$ & $-16.00 *$ \\
\hline EC-3 x EC-5 & 3.00 & 7.14 & -3.23 & 0.51 & $70.00 *$ & $-10.53^{*}$ & 3.90 & 0.00 & $-22.00 *$ \\
\hline EC-3 x GP-210 & 1.93 & -6.45 & $-37.63^{*}$ & 0.35 & 16.67 & $-38.60^{*}$ & 2.80 & 0.00 & $-44.00 *$ \\
\hline EC-3 x GP-211 & 3.10 & $60.34^{*}$ & 0.00 & 0.59 & $96.67 *$ & 3.51 & 5.50 & $96.43^{*}$ & $10.00 *$ \\
\hline EC-3 x GP-141 & 2.50 & 0.00 & $-19.35^{*}$ & 0.41 & 2.50 & $-28.07 *$ & 5.07 & 5.56 & 1.33 \\
\hline EC-4 x EC-5 & 3.13 & 4.44 & 1.08 & 0.32 & 6.67 & $-43.86^{*}$ & 3.30 & $-15.38 *$ & $-34.00 *$ \\
\hline EC-4 x GP-210 & 2.60 & $-13.33^{*}$ & $-16.13^{*}$ & 0.31 & 3.33 & $-45.61^{*}$ & 3.20 & 1.91 & $-36.00 *$ \\
\hline EC-4 x GP-211 & 3.13 & 4.44 & 1.08 & 0.29 & -3.33 & $-49.12 *$ & 4.10 & $30.57^{*}$ & $-18.00 *$ \\
\hline EC-4 x GP-141 & 3.20 & 6.67 & 3.23 & 0.39 & -2.50 & $-31.58^{*}$ & 5.00 & 4.17 & 0.00 \\
\hline EC-5 x GP-210 & 3.00 & 7.14 & -3.23 & 0.57 & $91.11 *$ & 0.58 & 5.60 & $43.59 *$ & $12.00 *$ \\
\hline EC-5 x GP-211 & 3.03 & 8.33 & -2.15 & 0.56 & $86.67 *$ & -1.75 & 5.30 & $35.90 *$ & 6.00 \\
\hline EC-5 x GP-141 & 3.17 & $13.10^{*}$ & 2.15 & 0.31 & $-22.50 *$ & $-45.61 *$ & 5.20 & 8.33 & 4.00 \\
\hline $\begin{array}{l}\text { GP-210 x GP- } \\
211\end{array}$ & 2.10 & 1.61 & $-32.26^{*}$ & 0.33 & $65.00^{*}$ & $-42.11 *$ & 3.10 & $63.16^{*}$ & $-38.00 *$ \\
\hline $\begin{array}{l}\text { GP-210 x GP- } \\
141\end{array}$ & 3.10 & $24.00 *$ & 0.00 & 0.49 & $23.33^{*}$ & $-13.45^{*}$ & 5.00 & 4.17 & 0.00 \\
\hline $\begin{array}{l}\text { GP-211 x GP- } \\
141\end{array}$ & 2.43 & -2.67 & $-21.51^{*}$ & 0.43 & 8.33 & $-23.98^{*}$ & 5.10 & 6.25 & 2.00 \\
\hline $\begin{array}{l}\text { Abhijeet } \\
\text { (Check) }\end{array}$ & 3.10 & - & - & 0.57 & - & - & 5.00 & - & - \\
\hline S Ed \pm & 0.170 & & & 0.030 & & & 0.200 & & \\
\hline CD at $5 \%$ & 0.334 & & & 0.056 & & & 0.412 & & \\
\hline
\end{tabular}


Table.5 TSS, and fruit fly incidence, of parents, $\mathrm{F}_{1}$ 's and heterosis (over better parent and check) in muskmelon

\begin{tabular}{|c|c|c|c|c|c|c|}
\hline \multirow[b]{2}{*}{ Parents and $F_{1}$ 's } & \multicolumn{3}{|l|}{ TSS } & \multicolumn{3}{|c|}{ Fruit fly incidence } \\
\hline & $\begin{array}{c}\text { Mean } \\
(\%)\end{array}$ & $\begin{array}{l}\text { Per cent } \\
\text { heterosis } \\
\text { over BP }\end{array}$ & $\begin{array}{c}\text { Per cent } \\
\text { increase/decrease } \\
\text { over hybrid } \\
\text { Abhijeet }\end{array}$ & Mean & $\begin{array}{l}\text { Per cent } \\
\text { heterosis } \\
\text { over BP }\end{array}$ & $\begin{array}{c}\text { Per cent } \\
\text { increase/decrea } \\
\text { se over hybrid } \\
\text { Abhijeet }\end{array}$ \\
\hline Kesar & 11.60 & - & - & $8.67(17.08)$ & - & - \\
\hline EC-2 & 10.50 & - & - & $22.87(28.57)$ & - & - \\
\hline EC-3 & 12.50 & - & - & $32.10(34.49)$ & - & - \\
\hline EC-4 & 10.00 & - & - & $18.30(25.32)$ & - & - \\
\hline EC-5 & 12.00 & - & - & $24.60(29.72)$ & - & - \\
\hline GP-210 & 15.00 & - & - & $37.10(37.52)$ & - & - \\
\hline GP-211 & 15.00 & - & - & $35.20(36.38)$ & - & - \\
\hline GP-141 & 13.00 & - & - & $19.60(26.27)$ & - & - \\
\hline Kesar x EC-2 & 11.60 & 0.00 & -10.77 & $9.07(17.53)$ & 2.64 & $-25.66^{*}$ \\
\hline Kesar x EC-3 & 12.60 & 0.80 & -3.08 & $22.70(28.45)$ & $66.58^{*}$ & $20.65^{*}$ \\
\hline Kesar x EC-4 & 11.00 & -5.17 & $-15.38^{*}$ & $19.10(25.91)$ & $51.73^{*}$ & $9.88^{*}$ \\
\hline Kesar x EC-5 & 12.10 & 0.83 & -6.92 & $20.37(26.82)$ & $57.06^{*}$ & $13.74 *$ \\
\hline Kesar x GP-210 & 14.50 & -3.33 & 11.54 & $13.10(21.21)$ & $24.20^{*}$ & $-10.05^{*}$ \\
\hline Kesar x GP-211 & 15.00 & 0.00 & $15.38^{*}$ & 23.83(29.22) & 71.09* & 23.92* \\
\hline Kesar x GP-141 & 13.10 & 0.77 & 0.77 & $19.37(26.10)$ & $52.84^{*}$ & $10.69 *$ \\
\hline EC-2 x EC-3 & 12.70 & 1.60 & -2.31 & $24.60(29.72)$ & 4.04 & $26.04 *$ \\
\hline EC-2 $\times$ EC-4 & 10.00 & -4.76 & $-23.08^{*}$ & $19.10(25.91)$ & 2.32 & $9.88^{*}$ \\
\hline EC-2 x EC-5 & 11.90 & -0.83 & -8.46 & $19.73(26.34)$ & $-7.78^{*}$ & 11.70* \\
\hline EC-2 x GP-210 & 14.50 & -3.33 & 11.54 & $36.37(37.09)$ & $29.82 *$ & 57.29* \\
\hline EC-2 x GP-211 & 15.20 & 1.33 & $16.92 *$ & $25.03(29.99)$ & 4.99 & $27.18^{*}$ \\
\hline EC-2 x GP-141 & 11.90 & -8.46 & -8.46 & $20.23(26.73)$ & 1.73 & $13.36^{*}$ \\
\hline EC-3 x EC-4 & 12.50 & 0.00 & -3.85 & $21.67(27.73)$ & $9.52 *$ & $17.60^{*}$ \\
\hline EC-3 x EC-5 & 12.30 & -1.60 & -5.38 & $31.60(34.19)$ & $15.03 *$ & $45.00 *$ \\
\hline EC-3 x GP-210 & 15.00 & 0.00 & $15.38^{*}$ & $36.23(37.00)$ & $7.29 *$ & $56.91 *$ \\
\hline EC-3 x GP-211 & 14.20 & -5.33 & 9.23 & $12.83(20.98)$ & $-39.16^{*}$ & $-11.03 *$ \\
\hline EC-3 x GP-141 & 15.50 & $19.23^{*}$ & $19.23^{*}$ & $30.63(33.59)$ & $27.86^{*}$ & $42.45^{*}$ \\
\hline EC-4 x EC-5 & 11.00 & -8.33 & $-15.38^{*}$ & $24.37(29.57)$ & $16.77 *$ & $25.40 *$ \\
\hline EC-4 x GP-210 & 13.00 & $-13.33^{*}$ & 0.00 & $34.93(36.23)$ & $43.06^{*}$ & $53.65^{*}$ \\
\hline EC-4 x GP-211 & 13.80 & -8.00 & 6.15 & $30.83(33.71)$ & $33.11^{*}$ & 42.96* \\
\hline EC-4 x GP-141 & 12.20 & -6.15 & -6.15 & $18.83(25.72)$ & 1.55 & $9.08^{*}$ \\
\hline EC-5 x GP-210 & 14.30 & -4.67 & 10.00 & $23.97(29.29)$ & -1.45 & 24.22* \\
\hline EC-5 x GP-211 & 14.00 & -6.67 & 7.69 & $19.47(26.18)$ & $-11.91 *$ & $11.03^{*}$ \\
\hline EC-5 x GP-141 & 12.50 & -3.85 & -3.85 & $21.87(27.87)$ & $6.09^{*}$ & $18.19 *$ \\
\hline $\begin{array}{l}\text { GP-210 x GP- } \\
211\end{array}$ & 15.70 & 4.67 & $20.77 *$ & $29.57(32.92)$ & $-9.53^{*}$ & $39.61 *$ \\
\hline $\begin{array}{l}\text { GP-210 x GP- } \\
141\end{array}$ & 15.10 & 0.67 & $16.15^{*}$ & $30.67(33.62)$ & $27.98^{*}$ & 42.58* \\
\hline $\begin{array}{l}\text { GP-211 x GP- } \\
141\end{array}$ & 14.00 & -6.67 & 7.69 & $25.97(30.62)$ & $16.53^{*}$ & $29.86 *$ \\
\hline $\begin{array}{l}\text { Abhijeet } \\
\text { (Check) }\end{array}$ & 13.00 & - & - & $16.00(23.58)$ & - & - \\
\hline S Ed \pm & 0.850 & & & 1.060 & - & - \\
\hline CD at $5 \%$ & 1.696 & & & 2.108 & - & - \\
\hline
\end{tabular}


Table.6 Estimates of general combining ability effects of parents for different characters in muskmelon

\begin{tabular}{|c|c|c|c|c|c|c|c|c|c|c|c|}
\hline Parents & $\begin{array}{l}\text { Vine } \\
\text { length } \\
(\mathrm{m})\end{array}$ & $\begin{array}{c}\text { Days to } \\
\text { first } \\
\text { fruit } \\
\text { harvest }\end{array}$ & $\begin{array}{c}\text { Fruit } \\
\text { weight } \\
\text { (kg) }\end{array}$ & $\begin{array}{c}\text { Number of } \\
\text { marketable } \\
\text { fruits/hill }\end{array}$ & $\begin{array}{l}\text { Yield/ } \\
\text { hill } \\
\text { (kg) }\end{array}$ & $\begin{array}{c}\text { Size of } \\
\text { seed } \\
\text { cavity } \\
(\mathrm{cm})\end{array}$ & $\begin{array}{l}\text { Rind } \\
\text { thickness } \\
(\mathrm{cm})\end{array}$ & $\begin{array}{c}\text { Flesh } \\
\text { thicknes } \\
\text { s(cm) }\end{array}$ & $\begin{array}{l}\text { TSS } \\
(\%)\end{array}$ & $\begin{array}{l}\text { Shelf life } \\
\text { (days) }\end{array}$ & $\begin{array}{l}\text { Incidence } \\
\text { of fruit fly }\end{array}$ \\
\hline Kesar & $-0.24 * *$ & $-1.20 * *$ & $0.03 * *$ & $-0.44 * *$ & $-0.24 * *$ & $-0.24 * *$ & $0.09 * *$ & $0.51 * *$ & $-0.46^{*}$ & $0.77 * *$ & $-5.20 * *$ \\
\hline EC-2 & $-0.10 * *$ & $2.09 * *$ & $0.05^{* *}$ & $-0.54 * *$ & $-0.19 * *$ & $-0.48 * *$ & $0.02 *$ & $0.34 * *$ & $-0.89 * *$ & $0.59 * *$ & $-1.10 * *$ \\
\hline EC-3 & $-0.12^{* *}$ & $-3.22 * *$ & $0.09 * *$ & $-0.17 *$ & $0.39 * *$ & $0.59 * *$ & 0.01 & $-0.26 * *$ & 0.21 & $-0.38 * *$ & $1.92 * *$ \\
\hline EC-4 & $-0.18 * *$ & $1.53 * *$ & $-0.13 * *$ & 0.11 & $-0.54 * *$ & $-0.23 * *$ & $-0.05 * *$ & 0.06 & $-1.42 * *$ & $-0.46 * *$ & $-0.60 * *$ \\
\hline EC-5 & $-0.33 * *$ & $1.82 * *$ & $0.11^{* *}$ & $-0.68 * *$ & 0.03 & $0.28 * *$ & 0.00 & $0.10 *$ & $-0.56 * *$ & 0.05 & -0.17 \\
\hline GP-210 & $0.50 * *$ & $3.53 * *$ & $-0.19 * *$ & $1.53 * *$ & 0.10 & $0.17 * *$ & $-0.05 * *$ & $-0.46^{* *}$ & $1.44 * *$ & $-0.73 * *$ & $4.10 * *$ \\
\hline GP-211 & $0.38 * *$ & $-2.45 * *$ & $0.02 *$ & $0.30 * *$ & $0.47 * *$ & $-0.12 *$ & $-0.03 * *$ & $-0.23 * *$ & $1.42 * *$ & $-0.33 * *$ & $1.50 * *$ \\
\hline GP-141 & $0.09 * *$ & $-2.09 * *$ & $0.02 *$ & -0.12 & -0.04 & 0.02 & 0.01 & -0.06 & 0.26 & $0.49 * *$ & $-0.46^{*}$ \\
\hline $\operatorname{SE}\left(g_{i}\right) \pm$ & 0.03 & 0.45 & 0.01 & 0.07 & 0.06 & 0.06 & 0.01 & 0.04 & 0.18 & 0.04 & 0.22 \\
\hline
\end{tabular}

* Significant at $\mathrm{p}=0.05 ; * *$ Significant at $\mathrm{p}=0.01$ 
Table.7 Estimates of specific combing ability effects of $\mathrm{F}_{1}$ 's for different characters in muskmelon

\begin{tabular}{|c|c|c|c|c|c|c|c|c|c|c|c|}
\hline$F_{1}$ 's & $\begin{array}{l}\text { Vine } \\
\text { length } \\
\text { (m) }\end{array}$ & $\begin{array}{l}\text { Days to } \\
\text { first } \\
\text { fruit } \\
\text { harvest }\end{array}$ & $\begin{array}{l}\text { Fruit } \\
\text { weight } \\
\text { (kg) }\end{array}$ & $\begin{array}{l}\text { Number of } \\
\text { marketable } \\
\text { fruits/hill }\end{array}$ & $\begin{array}{l}\text { Yield/hill } \\
\text { (kg) }\end{array}$ & $\begin{array}{l}\text { Size of } \\
\text { seed } \\
\text { cavity } \\
(\mathrm{cm})\end{array}$ & $\begin{array}{l}\text { Rind } \\
\text { thickness }\end{array}$ & $\begin{array}{l}\text { Flesh } \\
\text { thickness }\end{array}$ & TSS & $\begin{array}{l}\text { Shelf } \\
\text { life }\end{array}$ & $\begin{array}{l}\text { Incidence } \\
\text { of fruit } \\
\text { fly }\end{array}$ \\
\hline Kesar x EC-2 & 0.04 & $-2.90 *$ & $0.14 * *$ & $0.73 * *$ & $1.62 * *$ & $-1.07 * *$ & $0.06 * *$ & $0.37 * *$ & -0.13 & $0.62 * *$ & $-5.22 * *$ \\
\hline Kesar x EC-3 & $-0.25 * *$ & -1.79 & 0.01 & 0.06 & 0.14 & $0.86 * *$ & 0.00 & -0.06 & -0.23 & -0.02 & $2.68 * *$ \\
\hline Kesar x EC-4 & -0.06 & -1.01 & 0.05 & $0.75 * *$ & $1.07 * *$ & 0.19 & $0.04 *$ & 0.12 & -0.20 & $0.46 * *$ & $2.66 * *$ \\
\hline Kesar x EC-5 & $-0.17 *$ & $4.87 * *$ & $0.08 * *$ & 0.04 & $0.40 *$ & $0.47 *$ & 0.02 & -0.02 & 0.04 & $-0.34^{*}$ & $3.15 * *$ \\
\hline Kesar x GP-210 & $0.58 * *$ & $6.32 * *$ & $-0.09 * *$ & 0.34 & $-0.37 *$ & 0.29 & 0.02 & $-0.23^{*}$ & 0.44 & $1.43 * *$ & $-6.73 * *$ \\
\hline Kesar x GP-211 & $0.35 * *$ & -1.66 & -0.01 & $0.81 * *$ & 0.06 & 0.17 & 0.02 & 0.10 & 0.96 & 0.24 & $3.88 * *$ \\
\hline Kesar x GP-141 & 0.15 & $-3.22 *$ & $0.09 * *$ & -0.08 & $0.57 * *$ & 0.30 & $0.04 *$ & $0.40 * *$ & 0.22 & $-0.29 *$ & $2.72 * *$ \\
\hline EC-2 x EC-3 & -0.05 & 2.12 & $0.18 * *$ & -0.37 & $0.49 * *$ & $0.90 * *$ & -0.03 & $1.01 * *$ & 0.30 & $0.26^{*}$ & -0.15 \\
\hline EC-2 x EC-4 & -0.10 & -1.74 & 0.00 & 0.00 & 0.02 & $0.59 * *$ & $0.07 * *$ & $-0.71 * *$ & -0.77 & 0.14 & $-1.44 *$ \\
\hline EC-2 x EC-5 & -0.04 & -0.62 & $0.06^{*}$ & $-0.52 *$ & $-0.45^{*}$ & $0.61 * *$ & 0.00 & $-0.58 * *$ & 0.27 & -0.06 & $-1.44 *$ \\
\hline EC-2 x GP-210 & $0.16^{*}$ & -0.90 & -0.01 & 0.16 & 0.28 & -0.08 & 0.00 & $-0.76 * *$ & 0.87 & $0.51 * *$ & $5.04 * *$ \\
\hline EC-2 x GP-211 & -0.09 & 1.08 & $-0.15 * *$ & $0.91 * *$ & 0.01 & 0.11 & 0.01 & -0.03 & $1.59 * *$ & $0.52 * *$ & 0.55 \\
\hline EC-2 x GP-141 & -0.10 & -0.38 & -0.05 & -0.30 & $-0.48 * *$ & 0.21 & 0.03 & $-0.70 * *$ & -0.55 & $-0.41 * *$ & -0.76 \\
\hline EC-3 x EC-4 & 0.02 & -1.02 & $0.06^{*}$ & $1.46^{* *}$ & $1.84 * *$ & $-0.45^{*}$ & $0.10 * *$ & -0.18 & 0.63 & $0.51 * *$ & $-2.64 * *$ \\
\hline EC-3 x EC-5 & $0.41 * *$ & $-5.41 * *$ & $0.13 * *$ & 0.27 & $0.97 * *$ & $-0.70 * *$ & $0.08 * *$ & $0.25^{*}$ & -0.43 & $-0.30 *$ & $3.39 * *$ \\
\hline EC-3 x GP-210 & 0.11 & 1.51 & $-0.09 * *$ & $1.17 * *$ & $0.40^{*}$ & 0.15 & -0.03 & $-0.26^{*}$ & 0.27 & $-0.62 * *$ & $1.93 * *$ \\
\hline EC-3 x GP-211 & 0.14 & -2.17 & $0.11 * *$ & 0.19 & $1.03 * *$ & $-0.96 * *$ & $0.19 * *$ & $0.67 * *$ & -0.51 & $1.68 * *$ & $-11.49 * *$ \\
\hline EC-3 x GP-141 & $0.16^{*}$ & $-3.10 *$ & -0.01 & -0.08 & -0.06 & -0.26 & -0.03 & -0.10 & $1.95 * *$ & $0.42 * *$ & $3.09 * *$ \\
\hline EC-4 x EC-5 & $-0.16^{*}$ & -0.53 & -0.01 & $-0.58 *$ & $-0.60 * *$ & 0.20 & $-0.06 * *$ & 0.07 & -0.10 & $-0.82 * *$ & 1.29 \\
\hline EC-4 x GP-210 & $0.29 * *$ & -1.48 & -0.01 & $0.68 * *$ & 0.13 & $-1.39 * *$ & -0.02 & 0.09 & -0.10 & -0.14 & $3.68 * *$ \\
\hline EC-4 x GP-211 & $0.40 * *$ & -2.09 & 0.03 & -0.29 & -0.14 & 0.06 & $-0.06 * *$ & $0.39 * *$ & 0.72 & $0.36 * *$ & $3.76 * *$ \\
\hline EC-4 x GP-141 & 0.11 & 2.44 & 0.03 & $-0.46^{*}$ & -0.23 & 0.03 & 0.01 & $0.29 *$ & 0.28 & $0.44 * *$ & $-2.27 * *$ \\
\hline EC-5 x GP-210 & $0.29 * *$ & -2.47 & $-0.09 * *$ & 0.38 & 0.06 & 0.36 & $0.19 * *$ & $0.45 * *$ & 0.34 & $1.75 * *$ & $-3.68 * *$ \\
\hline EC-5 x GP-211 & $0.34 * *$ & -1.68 & $0.06^{*}$ & $0.70 * *$ & $1.29 * *$ & $-0.75^{* *}$ & $0.16^{* *}$ & $0.25^{*}$ & 0.06 & $1.06 * *$ & $-4.19 * *$ \\
\hline EC-5 x GP-141 & $0.23 * *$ & 0.76 & -0.01 & 0.25 & 0.30 & 0.11 & $-0.12 * *$ & 0.21 & -0.28 & 0.13 & -0.54 \\
\hline GP-210 x GP-211 & -0.15 & $-3.69 * *$ & $0.15^{* *}$ & $-0.75 * *$ & $0.52 * *$ & 0.26 & -0.02 & -0.13 & -0.24 & $-0.37 * *$ & $-1.73^{*}$ \\
\hline GP-210 x GP-141 & -0.05 & -2.46 & 0.00 & 0.12 & 0.23 & -0.37 & $0.11 * *$ & $0.70 * *$ & 0.32 & $0.71 * *$ & 0.94 \\
\hline GP-211 x GP-141 & -0.13 & -2.17 & -0.03 & $-0.57 *$ & $-0.64 * *$ & 0.21 & 0.03 & -0.20 & -0.76 & $0.41 * *$ & 0.53 \\
\hline $\operatorname{SE}\left(s_{i j}\right) \pm$ & 0.08 & 1.39 & 0.03 & 0.22 & 0.18 & 0.19 & 0.02 & 0.11 & 0.55 & 0.13 & 0.68 \\
\hline
\end{tabular}


The parents GP-211 and EC-3 exhibited highly significant positive gca showing that these genotypes are good combiners. Eleven $F_{1}$ 's showed significantly positive sca effects.

Small seed cavity would mean more flesh thickness, which is desirable in muskmelon. Only three crosses, out of 28 showed significant negative heterosis, which is desirable. The crosses viz., Kesar x EC-2 ($21.82 \%)$ and EC-4 x GP-210 (-15.76\%) had shown significantly negative heterosis over hybrid Abhijeet. The poor heterosis for this character has been reported by Dixit and Kalloo (1984) and Kalb and Davis (1984a). These crosses (EC-4 x GP-210 and Kesar x EC-2) also showed highest negative sca effects, which is desirable for this trait. These F1's derived from good $x$ poor and good $x$ good combiner parents, indicating dominance and additive type of gene action. Rind thickness directly contributes towards keeping quality of the fruit. It becomes more important when one has to transport muskmelon to the distant market. The increase in rind thickness over better parent was found to be 96.67 per cent in cross EC-3 $x$ GP-211. Eleven $F_{1}$ 's showed significantly positive heterosis over better parent. Similar findings have also been reported earlier (Semerjiev,1977, Nandpuri et al., 1982, Kalb and Davis,1984a, and Kitroongruang et al.,1992 and Choudhary 2003). Parents Kesar and EC-2 were found good general combiners for rind thickness. The crosses, EC-3 x GP211 and EC-5 x GP-210 had highest sca effects. Therefore, these could be exploited for improvement in rind thickness.

Flesh thickness is one, which contributes greatly towards yield in muskmelon. In the present study, the appreciable amount of heterobeltiosis was noticed. The per cent increase in heterosis over better parent was found to be 60.34 per cent. It is appreciable that five crosses had shown significantly higher flesh thickness over hybrid Abhijeet. Poor to medium level of heterosis for this trait has been reported by some researcher (Lippert and Hall, 1972a; Lippert and Legg, 1972a; Chadha and Nandpuri, 1980b; More and Seshadri, 1980; Kalb and Davis, 1984a; Randhawa and Singh 1990; Kitroongruang et al., 1992; Munshi and Verma, 1999 and Choudhary 2002). Both gca and sca effects were found to be significant. Ten $F_{1}$ 's had shown significantly positive sca effects. The crosses EC-2 x EC-3 and GP-210 x GP-141 had highest sca effects. Since muskmelon is a dessert fruit, TSS content of fruit is highly valued especially in view of consumer's preference. The range for heterosis over better parent for TSS was from -13.33 to $19.23 \%$. Only cross, EC-3 x GP-141 had shown significantly positive heterobeltiosis. Hence, most of the experimental $F_{1}$ 's did not reveal positive heterosis over the better parents, conforming that the character was primarily under the control of additive genes (Chadha $e t$ al.1972). GP-210 and GP-211 were the best general combiners. Crosses EC-3 x GP-141 and EC-2 $x$ GP-211 exhibited highly significant positive sca effects.

Shelf life is also an important trait to increase the transportability of fruits. It is contributed by many factors such as thickness of rind, toughness of skin, netting of skin, thickness of flesh and size of seed cavity. This however, may not be a very precise conclusion because it does not take into account the biochemistry, which goes into softening of the fruits. Out of $28 \mathrm{~F}_{1}$ 's, nine and five crosses revealed significantly longer shelf life over better parent and standard check, respectively. Maximum heterosis over better parent was reported in the cross EC-3 x GP-141. The results are quite close to those of Foster (1967), Semerjiev (1977) and Nandpuri et al., (1982). Cross EC-5 x GP-210 (1.75) had highest positive sca effect. This cross was derived from poor $\mathrm{x}$ good combiners 
indicating dominance $\mathrm{x}$ additive type of gene interaction.

The crosses with high per se performance, high sca effects and involving good $\mathrm{x}$ good combiner could be frequently advanced to obtain high frequency of transgressive segregants and simple pedigree method may give desirable results. However, for crosses involving good $\mathrm{x}$ poor or poor $\mathrm{x}$ poor general combiners, biparental mating, dialles selective mating (Jensen, 1970) and recurrent selection procedure is needed.

Since, most of the crosses had shown significant heterosis over their respective better parent and also over standard hybrid "Abhijeet", exploitation of heterosis by developing hybrid varieties is therefore advocated.

\section{References}

Chadha, M.L. and Nandpuri, K.S. 1977. Estimation of top cross performance in some muskmelon (Cucumis melo L.) varieties. Indian J. Hort. 34(1): 40-43.

Chadha, M.L. and Nandpuri, K.S. 1980a. Combining ability studies in muskmelon (Cucumis melo L.). Indian J. Hort., 37(1): 55-61.

Chadha, M.L. and Nandpuri, K.S. 1980b. Hybrid vigour studies in muskmelon. Indian J. Hort., 37(3): 276-282.

Chadha, M.L., Nandpuri, K.S. and Singh, S. 1972. Inheritance of quantitative characters in muskmelon. Indian $J$. Hort., 29: 174-178.

Choudhary, B.R. 2002. Heterosis and combining ability studies in muskmelon (Cucumis melo L.). Ph. D. Thesis, S.K.N. College of Agriculture (RAU), Jobner.

Choudhary, B.R., Dhaka, R.S. and Fageria, M.S. 2003. Heterosis for yield and yield related attributes in muskmelon
(Cucumis melo L.). Indian J. Genet, 63(1): 91-92.

Choudhary, B.R., Fageria, M.S. and Dhaka, R.S. 2004. Correlation and path coefficient analysis in muskmelon (Cucumis melo L.). Indian J. Hort., 61(2): 158-162.

Choudhary, M.L. and Mandal, G. 1987. Correlation and path analysis in cucumber (Cucumis sativus L.). Haryana J. Hort. Sci., 16(3-4): 269273.

Dhaliwal, M.S. 1997. Heterosis in muskmelon- a review. Agric. Reviews (1): 35-42.

Dhaliwal, M.S. and Lal, T. 1996. Genetics of some important characters using line $\mathrm{x}$ tester analysis in muskmelon. Indian J. Genet. 56(2): 207-213.

Dhaliwal, M.S., Lal, T. and Dhiman, J.S. 1996. Character association and causation in muskmelon. Indian $J$. Agric. Res. 30(2): 80-84.

Dixit, J. and Kalloo, G. 1983. Heterosis in muskmelon (Cucumis melo L.). J. Res. HAU 13(4): 549-553.

Fageria, M.S. and Luthra, J.P. 2005. Genetic variability in muskmelon (Cucumis melo L.). In: National Seminar on Cucurbits, 22-23 Sept. 2005, GBPUAT, Pantnagar, pp. 142.

Feyzian, E., Dehghani H., Rezai., A.M and Jalali Javaran M., 2009 Diallel cross analysis for maturity and yield-related traits in melon (Cucumis melo L.) Euphytica 168 (2): 215-223.

Fisher, R.A. and Yates, F. 1963. Statistical Tables for Biological, Agricultural and Medical Research. Oliver and Boyad, Edinburg, London.

Fonsceca, S. and Patterson, P.L. 1968. Hybrid vigour in seven parent diallel cross in common winter wheat (Triticum aestivum L.). Crop Sci. 8: 85-88.

Foster, R.E. 1967. $F_{1}$ hybrid muskmelon-I. Superior performance of selected 
hybrids. Proc. Amer. Soc. Hort. Sci. 91:390-395.

Griffing, J.B. 1956. Concept of general and specific combining ability in relation to diallel crossing system. Aust. J. Biol. Sci. 9:463-493.

Jensen, N.F. 1970. A diallel selective mating system for cereal breeding. Crop Sci. 10:629-635.

Kalb, T.J., II and Davis, D.W. 1984a. Evaluation of combining ability, heterosis and genetic variance for fruit quality characteristics in bush muskmelon. J. Amer. Soc. Hort. Sci. 109 (3): 411-415.

Kalb, T.J., II and Davis, D.W. 1984b. Evalution of combining ability, heterosis and genetic variance for yield, maturity and plant characteristics in bush muskmelon. $J$. Amer. Soc. Hort. Sci. 109 (3): 416419.

Kalloo, G., Baswana, K.S. and Sharma, N.K. 1993. Muskmelon: Hisar Madhur is early fruiting. Indian Hort., 38(2): 12.

Kalloo, G., Dixit, J. and Sidhu, A.S. 1982. Genetic divergence in muskmelon (Cucumis melo L.). Genet. Agrar., 36: 1-3.

Kalloo, G., Dixit, J. and Sidhu, A.S. 1982. Path coefficient analysis in muskmelon (Cucumis melo L.). Indian J. Hort., 39: 243-246.

Kalloo, G., Dixit, J. and Sidhu, A.S. 1983. Studies on genetic variability and character association in muskmelon (Cucumis melo L.). Indian J. Hort. 40(1-2): 79-85.

Kitroongruang, M., Poo-Swang, W. and Tokumasu, S. 1992. Evaluation of combining ability, heterosis and genetic advance for plant growth and fruit quality characteristics in Thaimelon (Cucumis melo L. var. acidulus Naud.). Scientia Horticultur, 50(1-2): 79-87.
Lippert, L.F. and Hall, M.O. 1972a. Hybrid vigour in muskmelon crosses. California Agricultur, 26(2): 12-14.

Lippert, L.F. and Hall, M.O. 1972b. Performance and combining ability of muskmelon varieties in a diallel cross. California Agriculture 26(2): 14-15.

Lippert, L.F. and Legg, P.D. 1972a. Appearance and quality characters in muskmelon fruit evaluated by a tencultivar diallel cross. J. Amer. Soc. Hort. Sci, 97: 84-87.

Lippert, L.F. and Legg, P.D. 1972b. Diallel analysis for yield and maturity characteristics in muskmelon cultivars. J. Amer. Soc. Hort. Sci., 97: 87-90.

McCreight, J.D., Nerson, H. and Grumet, R. 1993. Melon, Cucumis melo L. In G. Kalloo and B.O. Bergh (eds). Genetic Improvement of Vegetable Crops. Pp. 267-294. Pergamon Press, Oxford, New York.

Mishra, J.P. and Seshadri, V.S. 1988. Male sterility in muskmelon (Cucumis melo L.): I. Studies on combining ability. Indian J. Genet. 48(3): 347-354.

Mishra, J.P. and Seshadri, V.S. 1988. Male sterility in muskmelon (Cucumis melo L.). I. Studies on combining ability. Indian J. Genet. 48(3): 347-354.

More, T.A. and Seshadri, V.S. 1980. Studies on heterosis in muskmelon (Cucumis melo L.). Veg. Sci.7: 27-40.

More, T.A., Mishra, J.P., Seshadri, V.S., Doshi, S.P. and Sharma, J.C. 1987. Association of fruit shape with flesh area and flesh proportion in muskmelon. Ann. Agric. Res. 8(2): 237-242.

More, T.A., Seshadri, V.S., Doshi, S.P. and Sharma, J.C. 1980. Monoecious sex forms in muskmelon. Cucurbit Genetics Co-op. Repor, 3:32-33.

Munshi, A.D. and Verma, V.K. 1997. Studies on heterosis in muskmelon (Cucumis 
melo L.). Veg. Sci, 24(2): 103-106.

Munshi, A.D. and Verma, V.K. 1998. A note on gene action in muskmelon (Cucumis melo L.). Veg. Sci, 25(1): 93-94.

Munshi, A.D. and Verma, V.K. 1999. Combining ability in muskmelon (Cucumis melo L.). Indian J. Agric. Sci, 69(3): 214-216.

Nandpuri, K.S., Lal, T. and Dhiman, J.S. 1993. Punjab Rasila-high yielding and downy mildew tolerant variety of muskmelon. J. Res. PAU 30(1-2): 125.

Nandpuri, K.S., Lal, T. and Singh, S. 1983. Line $\mathrm{x}$ tester analysis of combining ability in muskmelon. J. Res. PAU 20: 29-38.

Nandpuri, K.S., Singh, S. and Lal, T. 1974a. Combining ability studies in muskmelon. J. Res. PAU 11(3): 225229.

Nandpuri, K.S., Singh, S. and Lal, T. 1974b. Study on the comparative performance of $\mathrm{F}_{1}$ hybrids in and their parents in muskmelon. J. Res. PAU 11(3): 230238.

Nandpuri, K.S., Singh, S. and Lal, T. 1975. Germplasm screening for the improvement of some economic characters in muskmelon (Cucumis melo L.). J. Res. PAU 12: 252-257.

Nandpuri, K.S., Singh, S. and Lal, T. 1982. Punjab Hybrid - a new variety of muskmelon. Prog. Fmg. 18: 3-4.

Panse, V.G. and Sukhatme, P.V. 1995. Statistical Methods for Agricultural
Workers. ICAR, New Delhi, India.

Randhawa, K.S. and Singh, M.J. 1990. Assessment of combining ability, heterosis and genetic variance for fruit quality characters in muskmelon (Cucumis melo L.). Indian J. Genet, 50(2): 127-130.

Semerjiev, P. 1977. The production of hybrid melons. From Abstracts of Bulgarian Scientific Literature, A., 22(3) Abst. No. 550.

Seshadri, V.S., Mishra, J.P., Sharma, J.C. and More, T.A. 1983. Heterosis breeding in melons - problems and prospects. South Indian Hort.,Commemoration issue): 74-82.

Shashikumar, K.T., and Pitchaimuthu, M. 2016. Heterosis and combining ability analysis of quantitative and qualitative traits in Muskmelon (Cucumis melo L.), 6 (2): 341-348.

Swamy, K.R.M. and Dutta, O.P. 1990. Inheritance of fruit dry matter content in muskmelon (Cucumis melo L.). Indian J. Agric. Res. 27(2): 87-95.

Swamy, K.R.M. and Dutta, O.P. 1993. Inheritance of fruit dry matter content in muskmelon (Cucumis melo L.). Indian J. Agric. Res. 27(2): 87-95.

Swamy, K.R.M., Dutta, O.P., Ramachander, P.R. and Wahi, S.D. 1985. Variability studies in muskmelon (Cucumis melo L.). Madras Agric. J. 72 (1): 1-5.

Swarup, V. 1991. Breeding Procedures for Cross-Pollinated Vegetable Crops. ICAR, New Delhi.

\section{How to cite this article:}

Saroj Rolania and Fageria, M.S. 2018. Heterosis and Combining Ability Evaluation for Yield, Quality, and Fruit Fly Resistance in Muskmelon. Int.J.Curr.Microbiol.App.Sci. 7(07): 902-915. doi: https://doi.org/10.20546/ijcmas.2018.707.109 